\title{
BMJ Open Social support network typologies and their association with dementia and depression among older adults in Singapore: a cross-sectional analysis
}

\author{
Ying Wen Lau, ${ }^{1}$ Janhavi Ajit Vaingankar, ${ }^{1}$ Edimansyah Abdin, ${ }^{1}$ Saleha Shafie, ${ }^{1}$ \\ Anitha Jeyagurunathan, ${ }^{1}$ Yunjue Zhang, ${ }^{1}$ Harish Magadi, ${ }^{2}$ Li Ling Ng, ${ }^{3}$ \\ Siow Ann Chong, ${ }^{1}$ Mythily Subramaniam ${ }^{1}$
}

To cite: Lau YW, Vaingankar JA, Abdin E, et al. Social support network typologies and their association with dementia and depression among older adults in Singapore: a crosssectional analysis. BMJ Open 2019;9:e025303. doi:10.1136/ bmjopen-2018-025303

- Prepublication history and additional material for this paper are available online. To view these files, please visit the journal online (http://dx.doi. org/10.1136/bmjopen-2018025303).

Received 9 July 2018 Revised 6 March 2019 Accepted 9 May 2019

Check for updates

(C) Author(s) (or their employer(s)) 2019. Re-use permitted under CC BY-NC. No commercial re-use. See rights and permissions. Published by BMJ.

${ }^{1}$ Research Division, Institute of Mental Health, Singapore, Singapore

${ }^{2}$ Department of Geriatric Psychiatry, Institute of Mental Health, Singapore, Singapore ${ }^{3}$ Psychological Medicine, Changi General Hospital, Singapore, Singapore

Correspondence to Dr Mythily Subramaniam; Mythily@imh.com.sg

\section{ABSTRACT}

Objectives To examine the social support network type and its associations with depression and dementia among older adults in Singapore.

Design This study is a cross-sectional analysis of data from the Well-being of the Singapore Elderly study. The Practitioner Assessment of Network Type was used to identify five social support network types. Odds Ratios (OR) of dementia and depression were estimated with logistic regression and multinomial logistic regression, respectively, adjusted for sociodemographic variables.

Setting Singapore.

Outcome measures 10/66 criteria and Automated Geriatric Examination for Computer Assisted Taxonomy computer algorithm.

Participants 2421 older adults aged 60 years and above, and their informants.

Results Logistic regression revealed that as compared with participants in the family dependent social support network type, those in the locally integrated social support network type were negatively associated with dementia. It was observed that it is the older adults' perception of the quality of social interaction that influences the likelihood of depression.

Conclusion The social support network typology presents knowledge about the older adults' social network profile and their cognitive functioning-ability which would help stakeholders better identify older adults who might be at risk of cognitive decline or experiencing delay in diagnosis of dementia.

\section{INTRODUCTION}

Population ageing is a worldwide demographic trend which has intensified in recent decades due to increasing life expectancies and declining fertility rates. ${ }^{1}$ Singapore is no exception to this phenomenon, where older adults over the age of 60 form $18.8 \%$ of the resident population. ${ }^{2}$ This percentage is expected to increase further as more baby boomers (ie, those born between 1947 and 1964) age. ${ }^{3}$ Consequently, the old-age support ratio is deteriorating. ${ }^{2}$ From the perspective
Strengths and limitations of this study

- This study was conducted in a multiethnic setting in Singapore and explores the association of social support network type with dementia and depression in older adults aged 60 and above.

- To our knowledge, this is the first study in Singapore that uses the Practitioner Assessment of Network Type to classify social support networks in older adults which allows for comparison with similar international studies.

- While we are unable to derive any causal inferences from our results given that this is a cross-sectional study, social support network type was found to be significantly associated with dementia.

of healthcare, this translates to increasing socioeconomic burden on the younger population to support and care for the population of older adults as the magnitude of comorbidity and disability in older adults intensify with age. ${ }^{45}$ It is, therefore, imperative that the older adults maintain a good state of health and well-being.

In the context of mental health, the most common neuropsychiatric disorders diagnosed in older adults are depression and dementia. ${ }^{6}$ Depression in the older adults has been established as a risk factor for mortality, poor health and disability ${ }^{7-10}$ and dementia is one of the major causes of disability in older adults ${ }^{11}$ ranked the ninth most burdensome condition in people aged 60 and above. ${ }^{12}$ In Singapore, the prevalence of dementia was $10 \%{ }^{13}$ and prevalence of depression was $3.7 \%^{14}$ among those aged 60 years and above. Due to the disabling effects, dementia and depression can hinder independent living in the older adults.

Apart from biological factors, social support network has been found to have a direct 
effect on the older adults' mental health. ${ }^{15}$ Social support network can generally be defined as the network of social interactions that provide help and companionship when in need. ${ }^{16-18}$ Studies have shown that social activities of the older adults are associated with depression ${ }^{19} 20$ and dementia. ${ }^{21}$ Maintaining sociability in old age is found to buffer unfavourable effects of ageing, some of which could also arise from depression and dementia, such as mortality, ${ }^{22}{ }^{23}$ institutionalisation, ${ }^{24}{ }^{25}$ functional decline ${ }^{26}$ and disability. ${ }^{27} 28$ In contrast, social disengagement brings about higher risks of morbidity ${ }^{29}{ }^{30}$ and cognitive decline. ${ }^{31}$ Therefore, understanding the social support network of older adults would help to facilitate the development of effective health policies and targeted interventions where necessary. ${ }^{32}$

Aspects of social support network such as network size, frequency of contact, living are usually examined individually. ${ }^{2028}$ However, Fiori $e t a l^{33}$ reasoned that it might be more informative to examine social support network typology instead of the individual aspects of social support network-social support network typology identifies categories of social network composition to capture distinct social environments that may be harmful or beneficial to health outcomes. ${ }^{34}$ On this premise, our study adopted Wenger's ${ }^{35}$ concept of social network typology, comprising five distinct social support network types each representing different levels of integration into the society and the extent of support received within the social network. This provides an overview into the state of the social support network rather than quantitative interpretations of the network.

To the best of our knowledge, no previous research conducted in Singapore has explored the association of social support network/socialsupport network typology with dementia and depression. Chan et als study ${ }^{20}$ which explored the association between social network and depression had found that weak social networks outside the household were associated with higher depressive symptoms scores. However, the social network was quantified by a total score and was meant to identify persons at risk of social isolation (weak social network) using a cut-off score instead of providing an insight into the condition of the social network. Considering the lack of studies on social support network typology among the older adults in Singapore and noting the association of social support networks with depression and dementia, this study aims to (1) examine the social support network typology in this population-based sample of older adult and (2) identify which social support network type is associated with depression and dementia to predict the group of older adults who may require interventions in the future. ${ }^{36}$

\section{MATERIALS AND METHODS}

\section{Study design}

The data used was from the Well-being of the Singapore Elderly (WiSE) study, a single-phase cross-sectional survey conducted in 2013. Participants of this study were Singapore residents and permanent residents aged 60 years and above, randomly selected from a national registry using a disproportionate stratified sampling design. Residents aged 75 years old and above and those of Malay and Indian ethnicity were oversampled to ensure reliability of our estimates. Participants who were in day care centres, nursing homes, and institutions were also included. All interviewers underwent a 2-week training on ethical guidelines and administration of the questionnaires. The survey was administered at the residence of the older adults through face-to-face interviews using an online computer-assisted personal interviewing application to both the participants and their chosen informants, defined as 'a person who knows the older person best' and could be a caregiver, co-residents or other close contacts. Written informed consent was obtained from all respondents or their legal representative/next-of-kin prior (if necessary) to the study. The methodology of the WiSE study can be found in an earlier article. ${ }^{13}$

\section{Patient and public involvement}

No patient or the public were involved in the development of the research question, study design and outcome measures. No patients or the public were involved in the recruitment to and conduct of the study. Results of the study were not disseminated to the participants directly but would be reported in publications and newsletters to the communities.

\section{Measures}

The WiSE study had adopted the $10 / 66$ protocol $^{37}$ developed by the 10/66 Dementia Research Group; the 10/66 questionnaire was considered to be suitable for use in the Singapore population given that it was developed principally for the lower-income and middle-income countries and had been validated in China, India and Malaysia. The entire test battery was administered to assess the participants. Measures used in this current study were from the 10/66 test battery.

Sociodemographic variables-age group, gender, ethnicity, marital status, employment status, education level, income level and number of assets were recorded as part of the survey. The income variable refers to the receipt of income, benefits, pensions or allowances and is analysed as a binary variable (yes/no). Participants were also asked from a list of household items if they owned any of these items and the positive responses was summed to derive the assets variable which can range from 0 to 8 .

The Practitioner Assessment of Network Type (PANT) is an eight-item instrument (see online supplementary text 1 ) used to identify the group of people whom the older adults rely on for support. The older adults 'responses to the questions were measured using the exact same algorithm developed by Wenger to identify the social support network typologies. ${ }^{35}$ The five different social network typologies (see online supplementary text 2) are: family dependent support network type, locally integrated support network type, locally self-contained 
support network type, wider community-focused support network type and private restricted support network type.

Dementia diagnosis was established using the 10/66 criteria,${ }^{37}$ and its algorithm was validated in our sample. ${ }^{13}$ The diagnosis requires ${ }^{38}$ : the Geriatric Mental State (GMS) examination, a semi-structured clinical mental state interview, which applies the Automated Geriatric Examination for Computer Assisted Taxonomy (AGECAT) computer algorithm (GMS-AGECAT) ${ }^{39}$; the Community Screening Instrument for Dementia (CSI-D) ${ }^{40}$ which incorporates the Consortium to Establish a Registry for Alzheimer's Dementia (CERAD) animal naming verbal fluency task and modified CERAD 10-word list learning task with delayed recall to generate the global cognitive score (COGSCORE) ${ }^{41}$ and; an informant interview, the CSI-D informant score (RELSCORE), ${ }^{40}$ for evidence of cognitive and functional decline. Data were collected from both participants and their informants. The cut-off point for dementia diagnosis was derived from the logistic regression equation developed in the 10/66 international pilot study. ${ }^{38}$ The 'dementia' variable was treated as a dichotomous variable.

The GMS-AGECAT generates nine diagnostic clusters and the depression cluster was used to identify depression cases. ${ }^{39}$ A GMS-AGECAT score of 3 or more is indicative of depression; a score of 1-2 indicates subsyndromal depression and a score of 0 indicates no depression. This measure has been used in our sample. ${ }^{14}$ The 'depression' variable was treated as a categorical variable.

Participants were asked to answer questions on twelve common physical impairments and were grouped into three categories: none, one to two and three or more impairments. The 12 physical impairments were: arthritis/rheumatism, eyesight problems, hearing difficulty/deafness, persistent cough, breathlessness/difficulty breathing/asthma, high blood pressure, heart trouble/angina, stomach/intestine problems, faints/ blackouts, paralysis/weakness or loss of one leg or arm, skin disorders and cancer. Disability was measured by the 12-item WHO Disability Assessment Schedule 2.0 $0^{42}$ and is a continuous variable where higher scores indicate greater disability.

\section{Statistical analysis}

All analyses were conducted using STATA V.14.0 (StataCorp, 2015). The data were weighted and analysed using survey data analysis procedures to adjust for oversampling, non-response and poststratification according to age and ethnicity. The sample was categorised into the social support network typologies. Descriptive analyses were conducted to describe sample characteristics for the overall sample and also for each social support network type. Logistic regression analysis was performed to examine the association between dementia and social support network typologies. Multinomial logistic regression was used to examine the association between depression and social support network typologies. Both regression analyses were controlled for sociodemographic characteristics including age, gender, ethnicity, education level, marital status, educational status and employment status, income, assets, as well as physical impairment and disability since these factors may influence the social support network typologies that an older adult is in. All statistically significant results were reported at $p$ value $<0.05$.

\section{RESULTS}

\section{Sociodemographic characteristics}

Data from 2421 older adult respondents and their informants were included in the analysis. Sociodemographic characteristics of the study sample are presented in table 1 . The majority of the participants were females $(57 \%)$, aged between 60 and 74 years $(74.8 \%)$, of Chinese ethnicity $(82.6 \%)$ and currently married (65.4\%). Majority of them $(89.3 \%)$ received some form of income (eg, salary, benefits, pensions, allowances) and had one or two physical impairments (51.7\%).

\section{Sociodemographic characteristics by social support network typologies}

Nearly half of the participants $(44.4 \%)$ belonged to the family dependent social support network type. $27.1 \%$ were in the locally integrated social support network type, $4.6 \%$ in the locally self-contained social support network type, $0.8 \%$ in the wider community-focused social support network type and $2.6 \%$ in the private social support network type. $20.4 \%$ of the participants were classified under non-conclusive network. Table 1 presents the sample characteristics for each social support network type. Univariate analyses ( $\chi^{2}$ test or analysis of variance) revealed significant differences in age group, ethnicity, education level, income, dementia and disability across the social support network typologies. The PANT characteristics of each social support network type may be found in online supplementary text 3 .

\section{Social support network typologies: association with dementia and other correlates}

Logistic regression revealed that dementia was negatively associated ( $\mathrm{OR}=0.34,95 \% \mathrm{CI} 0.15$ to 0.79$)$ with participants in the locally integrated social support network type as compared with those in the family dependent social support network type. There were no dementia cases identified in the wider community-focused social support network type. In addition, dementia was found to be negatively associated with Indian ethnicity and higher education level, and positively associated with age group of 75 years and above, those with no income and those with disability. The results are presented in table 2.

\section{Social support network typologies: association with depression and other correlates}

Multinomial logistic regression revealed that social support network typologies were not associated with depression. Depression and subsyndromal depression 


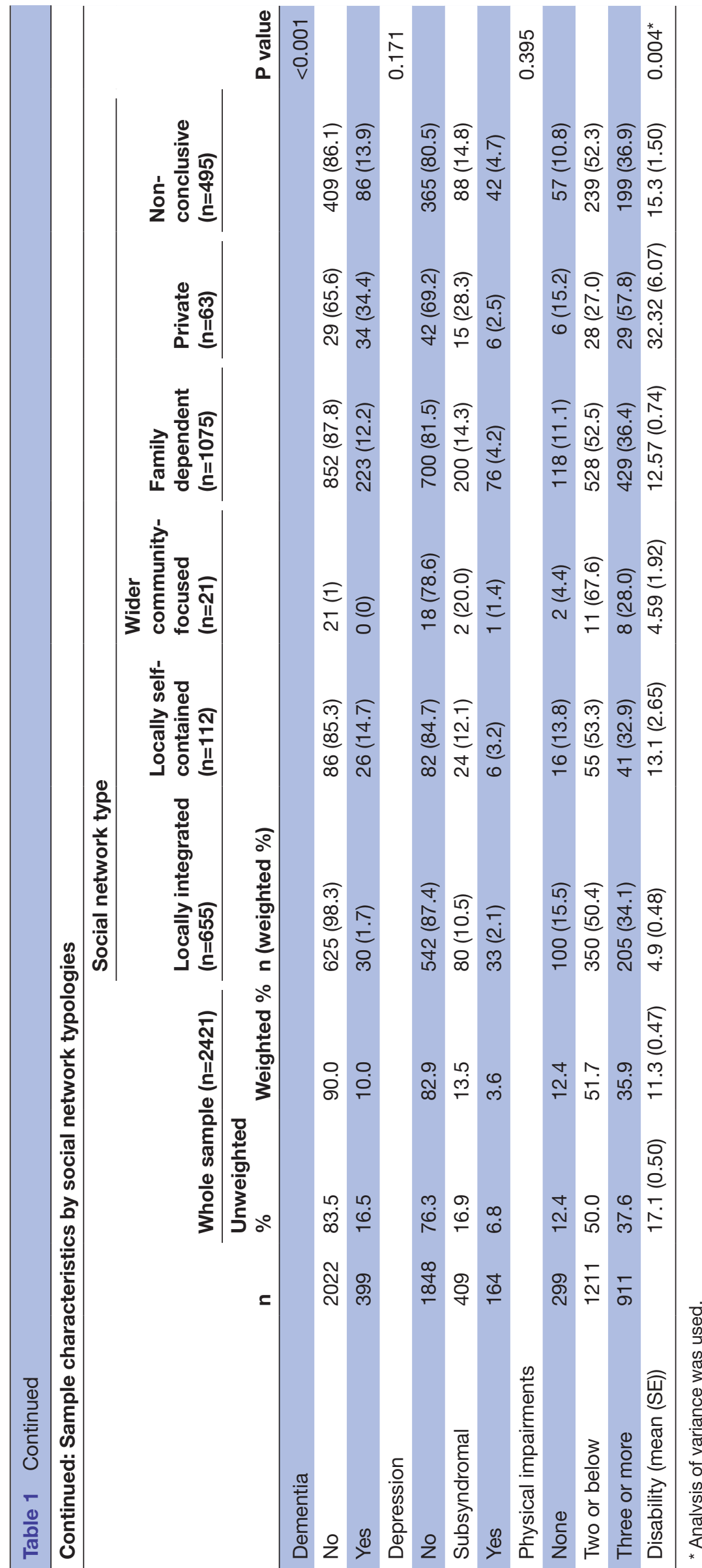

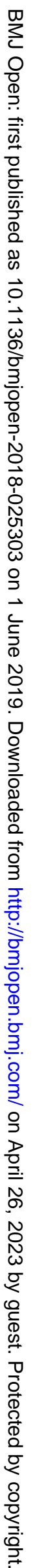


Table 2 Association between social network typologies and dementia

\begin{tabular}{|c|c|c|c|}
\hline & & OR & $95 \% \mathrm{Cl}$ \\
\hline \multirow[t]{6}{*}{ Social network type } & Family dependent & ref & \\
\hline & Locally integrated & $0.34^{*}$ & (0.15 to 0.79$)$ \\
\hline & Locally self-contained & 1.11 & (0.22 to 5.68$)$ \\
\hline & Wider community focused & No dementia cases & \\
\hline & Private & 1.15 & (0.36 to 3.73 ) \\
\hline & Non-conclusive & 0.80 & (0.43 to 1.51$)$ \\
\hline \multirow[t]{3}{*}{ Age group } & $60-74$ & ref & \\
\hline & $75-84$ & $3.64^{*}$ & (1.82 to 7.26$)$ \\
\hline & $85+$ & $6.92^{*}$ & (3.08 to 15.55$)$ \\
\hline \multirow[t]{2}{*}{ Sex } & Female & ref & \\
\hline & Male & 1.46 & (0.77 to 2.75$)$ \\
\hline \multirow[t]{4}{*}{ Ethnicity } & Chinese & ref & \\
\hline & Malay & 0.74 & (0.43 to 1.26$)$ \\
\hline & Indian & $0.52^{\star}$ & (0.30 to 0.90$)$ \\
\hline & Others & No dementia cases & \\
\hline \multirow[t]{4}{*}{ Marital status } & Married/cohabiting & ref & \\
\hline & Never married & 0.57 & (0.11 to 2.79$)$ \\
\hline & Widowed & 1.13 & (0.59 to 2.14$)$ \\
\hline & Divorced/separated & 0.45 & (0.13 to 1.49$)$ \\
\hline \multirow[t]{5}{*}{ Education } & Completed primary & ref & \\
\hline & None & 0.81 & (0.37 to 1.75$)$ \\
\hline & Did not complete primary & 0.79 & (0.38 to 1.64$)$ \\
\hline & Completed secondary & $0.36^{*}$ & (0.14 to 0.88$)$ \\
\hline & Completed tertiary & $0.13^{\star}$ & (0.03 to 0.63$)$ \\
\hline \multirow[t]{2}{*}{ Income } & Yes & ref & \\
\hline & No & $3.59^{\star}$ & (1.87 to 6.88$)$ \\
\hline \multirow[t]{3}{*}{ Physical impairments } & Two or below & ref & \\
\hline & None & 1.65 & (0.63 to 4.33$)$ \\
\hline & Three or more & 0.90 & (0.51 to 1.57$)$ \\
\hline Assets & & 0.90 & (0.74 to 1.11$)$ \\
\hline Disability & & $1.06^{*}$ & (1.05 to 1.08$)$ \\
\hline Constant & & $0.03^{\star}$ & (0.01 to 0.18$)$ \\
\hline
\end{tabular}

*Significant given $95 \% \mathrm{Cl}$ does not contain 1.

were found to be positively associated with the Malay and Indian ethnicity, physical impairments as well as disability. The results are presented in table 3 .

\section{DISCUSSION}

This study sought to examine the social support network typologies and its association with depression and dementia in a population-based sample of older adults in Singapore. Our results indicated that the social support network typology profile of the older adults in Singapore differed from those in other developed and developing countries. We also identified that being in the locally integrated social support network type was associated with lower odds of developing dementia as compared with being in the family dependent social support network type. On the contrary, we did not find any significant association between social support network typology and depression.

We observed that the nature of social interaction among the older adults in Singapore was unlike other countries. The findings from a cross-sectional study conducted in Ireland on 683 people aged 65-99years, revealed that older adults in Ireland belonged mostly to the locally integrated social network type. ${ }^{15}$ This was similar to the findings from population-based studies conducted on individuals aged 65 years and above in eight 
Table 3 Association between social network typologies and depression

\begin{tabular}{|c|c|c|c|c|}
\hline & & & OR & $95 \% \mathrm{Cl}$ \\
\hline \multirow[t]{15}{*}{ Depression } & Social network type & Family dependent & ref & \\
\hline & & Locally integrated & 0.62 & $(0.29$ to 1.31$)$ \\
\hline & & Locally self-contained & 0.56 & (0.15 to 2.14$)$ \\
\hline & & Wider community focused & 0.35 & (0.03 to 3.78$)$ \\
\hline & & Private & 0.31 & $(0.08$ to 1.17$)$ \\
\hline & & Non-conclusive & 0.82 & (0.43 to 1.55$)$ \\
\hline & Ethnicity & Chinese & ref & \\
\hline & & Malay & $4.11^{*}$ & (2.31 to 7.33$)$ \\
\hline & & Indian & $5.97^{*}$ & (3.51 to 10.19$)$ \\
\hline & & Others & 3.37 & (0.63 to 17.99$)$ \\
\hline & Physical impairments & Two or below & ref & \\
\hline & & None & $0.23^{*}$ & (0.08 to 0.65$)$ \\
\hline & & Three or more & 2.05 & (1.16 to 3.60$)$ \\
\hline & Disability & & $1.04^{*}$ & (1.03 to 1.06$)$ \\
\hline & Constant & & $0.02^{*}$ & (0.00 to 0.09$)$ \\
\hline \multirow[t]{22}{*}{ Subsyndromal } & Social network type & Family dependent & ref & \\
\hline & & Locally integrated & 0.73 & $(0.46$ to 1.15$)$ \\
\hline & & Locally self-contained & 0.68 & (0.33 to 1.42$)$ \\
\hline & & Wider community focused & 1.58 & (0.15 to 16.94$)$ \\
\hline & & Private & 1.43 & $(0.44$ to 4.71$)$ \\
\hline & & Non-conclusive & 0.86 & (0.55 to 1.36$)$ \\
\hline & Age group & $60-74$ & & \\
\hline & & $75-84$ & 0.67 & (0.44 to 1.03$)$ \\
\hline & & $85+$ & $0.36^{\star}$ & $(0.19$ to 0.70$)$ \\
\hline & Ethnicity & Chinese & ref & \\
\hline & & Malay & 1.59 * & (1.14 to 2.23$)$ \\
\hline & & Indian & $2.13^{*}$ & (1.54 to 2.94$)$ \\
\hline & & Others & 1.42 & (0.45 to 4.44$)$ \\
\hline & Marital status & Married/cohabiting & ref & \\
\hline & & Never married & 1.04 & (0.47 to 2.30$)$ \\
\hline & & Widowed & 1.20 & $(0.76$ to 1.90$)$ \\
\hline & & Divorced/separated & $2.41^{*}$ & (1.13 to 5.12$)$ \\
\hline & Physical impairments & Two or below & ref & \\
\hline & & None & $0.36^{*}$ & (0.18 to 0.72$)$ \\
\hline & & Three or more & $2.16^{\star}$ & (1.49 to 3.13$)$ \\
\hline & Disability & & 1.02 & (1.01 to 1.03$)$ \\
\hline & Constant & & $0.20^{*}$ & (0.08 to 0.51$)$ \\
\hline Normal & (Base outcome) & & & \\
\hline
\end{tabular}

Only variables with significant results are presented in the table.

*Significant given $95 \% \mathrm{Cl}$ does not contain 1.

countries (Cuba, Dominican Republic, Peru, Venezuela, Mexico, Puerto Rico, China and India). ${ }^{34}$ In particular, the older adults in developing countries such as Latin America, India and China (the urban populations were an exception where majority of the older adults belonged to the private social support network type possibly due to urbanisation) mostly belonged to the locally integrated social network type. This indicated that while majority of older adults in Singapore had close relationships with family and/or relatives, they connected less with friends 
and neighbours as compared with the older adults in the aforementioned countries.

Approximately $94 \%$ of the resident households in Singapore live in government-developed public housing or private condominiums, ${ }^{2}$ both of which place the residents in close proximity to one another and are optimum for social interaction and involvement. However, cultural influences on support seeking ${ }^{43}$ may have an impact on the older adults' social lifestyle that is, our sample of older adults might not be comfortable being close or divulging too much information to others apart from family members, resulting in generally minimal interaction with other people. Also, given the small geographical size and convenience of transportation in Singapore, the older adults might not recognise the need to maintain social relationships since they could easily get in touch and depend on family members for help and support in times of need.

Another potential contributing factor to the disparity could be the older adults' physical health status. Our findings revealed that (1) majority of the older adults had more than one physical impairment, (2) half of those who had ' 2 or below' and 'more than 3' impairments belonged to the family dependent social support network type and (3) the disability score was highest in the private social support network type followed by the family-dependent social support network type. It is thus possible that physical health influences the degree of sociability of an older adult likely due to the inconveniences brought about by the impairment(s) thereby restricting their network.

A considerable percentage of our participants did not belong to any particular social support network type and were categorised as 'non-conclusive'. Based on our observation, there is no distinctive trait among this group of participants for meaningful interpretation. Their social support network could have been in a state of flux or shifting from one network to another due to changes in their circumstances. ${ }^{17}$ Even so, we had included this group of participants in our analysis as late life is likely a period of transitions where the older adults experience major changes such as retirement and bereavement of their close ones, requiring time to adjust to a 'new' life which might affect their living arrangements and social interactivity. ${ }^{45}$ Further exploration will be required to identify the actual reasons.

Ageing trends have been a national interest and several ministerial committees have been formed to address issues that could arise from an ageing population in Singapore. ${ }^{46}$ The policies were targeted at securing wellbeing in old age and had placed emphasis on active ageing and social involvement for the older adults to maintain an active and engaged lifestyle. ${ }^{47}$ Despite the long-standingefforts to promote social integration, observation from our sample showed otherwise. It might be helpful to conduct an evaluation of the current policies in place to determine their effectiveness and facilitate the development of future strategies. Nevertheless, it is heartening to note that the Singapore government is invested in this subject matter, continuously working on new initiatives to promote active ageing; one of the latest initiatives to commence in 2020 is the "Community Networks for Seniors' scheme to help lonely older adults find new friends and strengthen the community care network. ${ }^{48}$

About the influence of social support network typology on the prevalence of dementia, our results revealed that being in the locally integrated social support network type was significantly associated with lower odds of developing dementia as compared with being in the family dependent social support network type. Although the results were not significant, being in the non-conclusive social support network type was associated with lower odds of having dementia, while being in the locally self-contained social support network type and private social network type were associated with higher odds of having dementia. Wenger had observed that older adults in the private or locally self-contained social support networks were more vulnerable to social isolation than those in the locally integrated and wider community-focused social support network. ${ }^{49} 50$

The findings of this study are consistent with that from a longitudinal study conducted among a community-based cohort on older adults in Sweden, which concluded that older adults without close social ties (contact with friends and/or relatives) and who were single and living alone had an increased risk of developing dementia. ${ }^{51}$ Our sample had no diagnosed cases of dementia in the wider community focused social support network type. As such, we postulate that in addition to family relation which is an important source of support, ${ }^{52}$ it may be also beneficial for older adults to maintain an appropriate level of interaction with friends and/or neighbours to keep themselves engaged with the social environment which would help sustain their cognitive function to prevent or delay dementia. ${ }^{53}$

Our findings did not reflect any association between the social support network types and depression, in contrast to two studies conducted among community-dwelling older adults in Singapore. Both studies had established negative associations between social support and depression. It is noted that the instruments used to measure social support were however different. In the study conducted by Chan $e t a l,{ }^{20}$ social support was examined through: (1) living arrangements (categorical variable) and (2) social network (continuous variable) measured by the Lubben's Social Network Scale-Revised that assessed the size of network outside the household, frequency of contact, closeness and perceived social support from this network. Li et a $\tilde{l}^{4}$ similarly examined: (1) living arrangements (categorical variable) and (2) perceived social support (continuous variable) with the Duke Social Support Index which comprised two constructs: social satisfaction and social interaction.

The PANT combines both living arrangements and social network into an entirety and measured 'facts' such as distance and frequency of contact, unlike the other two scales which included subjective questions, for example, 
'Do you feel...' The way the questions are phrased suggests that the type of social support network per se has no effect on the onset of depression, but it is whether the older adult perceives support from the network that has an influence over depression. There were also studies which did not find the predictive effect of social support on depression in older adults. ${ }^{55}$ Further research is certainly required to conclude this inference, considering there may be confounding factors such as the mental health condition, outlook on life, degree of self-reliance, personal preference that may affect the older adults' assessment on the social support received.

Interpretation of these findings should take into consideration several limitations. First, our results might not be representative of the entire population of older adults as the response rate was $65.6 \%$ and might have missed out on participants with more severe dementia or depression. Second, we were unable to identify any associations/traits in the non-conclusive network, which leaves this particular group of older adults neglected when they might actually require intervention or attention. Third, the non-significant finding for depression could be attributed to the small sample sizes $(n<5)$ in the subgroups, reflected in table 1. Lastly, given the cross-sectional design of the study, we are unable to derive any causal inferences from our results.

Notwithstanding these limitations, this study provides preliminary insight into the usage of PANT and development of future interventions with regards to prevention against dementia and depression in population of older adults in Singapore corresponding to their social support network typology, since no similar research has been conducted before. Our findings have shown that the social support network typology presents us the knowledge about the older adults' social network profile (living arrangements and social contacts) and accordingly, their cognitive functioning-ability which could have an impact on the onset of dementia. Using the PANT, stakeholders will be able to identify isolated groups of older adults who might be at risk of cognitive decline. Appropriate interventions could then be implemented to ensure that the group of older adults will be cared for and promote the importance of social interaction among them. We infer from our findings that the social support network is not a good enough predictor for depression; rather, it is the older adults' perception of the quality of social interaction that will influence the likelihood of depression. In this regard, future research is essential to measure the quality of social interaction and establish its associated factors in older adults in order to develop effective preventive measures against depression.

Contributors YWL analysed the data, interpreted the findings and wrote the manuscript. JAV provided input into the study protocol, was involved in conducting the study and provided intellectual input for the article. EA managed the data and provided intellectual input for the article. SS, AJ and YZ were involved in conducting the study and provided intellectual input for the article. HM and LLN provided intellectual inputs for the article. SAC provided intellectual input for the article and was the principal investigator of the WiSE Study. MS provided input into the study protocol, was involved in conducting the study and critically reviewed the article. All authors have read and approved the final manuscript.

Funding The WiSE study was supported by the Ministry of Health, Singapore and the Singapore Millennium Foundation of the Temasek Trust.

Competing interests None declared.

Patient consent for publication Not required.

Ethics approval Ethical approval was obtained from the National Healthcare Group Domain Specific Review Board and the SingHealth Centralised Institutional Review Board.

Provenance and peer review Not commissioned; externally peer reviewed.

Data sharing statement Data are not available for online access, however readers who wish to gain access to the data can write to the corresponding author MS at mythily@imh.com.sg with their requests. Access can be granted subject to the Institutional Review Board (IRB) and the research collaborative agreement guidelines. This is a requirement mandated for this research study by our IRB and funders.

Open access This is an open access article distributed in accordance with the Creative Commons Attribution Non Commercial (CC BY-NC 4.0) license, which permits others to distribute, remix, adapt, build upon this work non-commercially, and license their derivative works on different terms, provided the original work is properly cited, appropriate credit is given, any changes made indicated, and the use is non-commercial. See: http://creativecommons.org/licenses/by-nc/4.0/.

\section{REFERENCES}

1. United Nations Population Division, Department of Economic and Social Affairs. World Population Ageing. 2013. Accessed July 10, 2017 http://www.un.org/en/development/desa/population/ publications/ageing/WorldPopulationAgeingReport2013.shtml

2. Department of Statistics, Singapore. Population Trends. 2016 http:// www.singstat.gov.sg/docs/default-source/default-document-library/ publications/publications_and_papers/population_and_population_ structure/population2016.pdf (Accessed July 10, 2017).

3. Ministry of Community Development, Youth and Sports. BabyBoomers Survey. 2009 http://www.nas.gov.sg/archivesonline/data/ pdfdoc/20090116004/baby_boomer_survey_7jan09.pdf (Accessed December 18, 2018)

4. Rodriguez-Blazquez C, Forjaz MJ, Prieto-Flores ME, et al. Health status and well-being of older adults living in the community and in residential care settings: are differences influenced by age? Aging Ment Health 2012;16:884-91

5. Luthy $\mathrm{C}$, Cedraschi $\mathrm{C}$, Allaz AF, et al. Health status and quality of life: results from a national survey in a community-dwelling sample of elderly people. Qual Life Res 2015;24:1687-96.

6. World Health Organization. Fact Sheet Dementia. 2016 http://www. who.int/mediacentre/factsheets/fs362/en/ (accessed $10 \mathrm{Jul} 2017$ ).

7. Egede LE. Major depression in individuals with chronic medical disorders: prevalence, correlates and association with health resource utilization, lost productivity and functional disability. Gen Hosp Psychiatry 2007;29:409-16.

8. Fu CC, Lee YM, Chen JD. Association between depressive symptoms and twelve-year mortality among elderly in a rural community in Taiwan. J Formos Med Assoc 2003;102:234-9.

9. Han B. Depressive symptoms and self-rated health in communitydwelling older adults: a longitudinal study. J Am Geriatr Soc 2002;50:1549-56.

10. Lenze EJ, Rogers JC, Martire LM, et al. The association of latelife depression and anxiety with physical disability: a review of the literature and prospectus for future research. Am J Geriatr Psychiatry 2001;9:113-35

11. World Health Organization. Fact Sheet: Mental health and older adults. 2016 http://www.who.int/mediacentre/factsheets/fs381/en/ (accessed 10 Jul 2017).

12. Alzheimer's Disease International, World Alzheimer Report. The global impact of dementia - an analysis of prevalence, incidence, cost \& trends. 2015. Available at https://www.alz.co.uk/research/Worl dAlzheimerReport2015.pdf (accessed 10 Jul 2017).

13. Subramaniam $M$, Chong SA, Vaingankar JA, et al. Prevalence of Dementia in People Aged 60 Years and Above: Results from the WiSE Study. J Alzheimers Dis 2015;45:1127-38.

14. Subramaniam M, Abdin E, Sambasivam R, et al. Prevalence of Depression among Older Adults-Results from the Well-being of the Singapore Elderly Study. Ann Acad Med Singapore 2016;45:123-33. 
15. Drennan J, Treacy MP, Butler M, et al. Support networks of older people living in the community. Int J Older People Nurs 2008;3:234-42.

16. Kim O. Mediation effect of social support between ethnic attachment and loneliness in older Korean immigrants. Res Nurs Health 1999;22:169-75.

17. Wenger GC. Support Networks of Older People: A Guide for Practitioners. Bangor: CSPRD, University of Wales, 1994.

18. Wenger GC, Keating N. Care network outcomes for the oldest old. CSPRD, Institute of Medical and Social Care Research. Bangor, UK: University of Wales, 2002.

19. A. Thiyagarajan J, Prince M, Webber M. Social support network typologies and health outcomes of older people in low and middle income countries - A 10/66 Dementia Research Group populationbased study. International Review of Psychiatry 2014;26:476-85.

20. Chan A, Malhotra C, Malhotra R, et al. Living arrangements, social networks and depressive symptoms among older men and women in Singapore. Int J Geriatr Psychiatry 2011;26:630-9.

21. Fratiglioni L, Paillard-Borg S, Winblad B. An active and socially integrated lifestyle in late life might protect against dementia. Lancet Neurol 2004;3:343-53

22. Nyqvist F, Pape B, Pellfolk T, et al. Structural and Cognitive Aspects of Social Capital and All-Cause Mortality: A Meta-Analysis of Cohort Studies. Soc Indic Res 2014;116:545-66.

23. Rasulo D, Christensen K, Tomassini C. The influence of social relations on mortality in later life: a study on elderly Danish twins. Gerontologist 2005;45:601-8.

24. Colantonio A, Kasl SV, Ostfeld AM, et al. Psychosocial predictors of stroke outcomes in an elderly population. J Gerontol 1993;48:S261-S268.

25. Steinbach U. Social networks, institutionalization, and mortality among elderly people in the United States. J Gerontol 1992;47:S183-S190.

26. Stuck AE, Walthert JM, Nikolaus T, et al. Risk factors for functional status decline in community-living elderly people: a systematic literature review. Soc Sci Med 1999;48:445-69.

27. Avlund $\mathrm{K}$, Lund $\mathrm{R}$, Holstein $\mathrm{BE}$, et al. Social relations as determinant of onset of disability in aging. Arch Gerontol Geriatr 2004;38:85-99.

28. Escobar-Bravo MÁ, Puga-González D, Martín-Baranera M. Protective effects of social networks on disability among older adults in Spain. Arch Gerontol Geriatr 2012;54:109-16.

29. Institute of Medicine. The Second Fifty Years: Promoting Health and Preventing Disability. Washington, DC: The National Academies Press, 1992. Available at. https://www.nap.edu/read/1578/chapter/1 (accessed 12 Jul 2017).

30. Tomaka J, Thompson S, Palacios R. The relation of social isolation, loneliness, and social support to disease outcomes among the elderly. J Aging Health 2006;18:359-84.

31. Barnes LL, Mendes de Leon CF, Wilson RS, et al. Social resources and cognitive decline in a population of older African Americans and whites. Neurology 2004;63:2322-6.

32. Keating $\mathrm{N}$, Otofonowski $\mathrm{P}$, Wenger $\mathrm{C}$, et al. Understanding the caring capacity of informal networks of frail seniors: a case for care networks. Ageing Soc 2003;23:115-27.

33. Fiori KL, Antonucci TC, Cortina KS. Social network typologies and mental health among older adults. J Gerontol B Psychol Sci Soc Sci 2006;61:P25-P32.

34. Santini ZI, Koyanagi A, Tyrovolas S, et al. Social network typologies and mortality risk among older people in China, India, and Latin America: A 10/66 Dementia Research Group population-based cohort study. Soc Sci Med 2015;147:134-43.
35 Wenger GC. A network typology: From theory to practice. J Aging Stud 1991:5:147-62.

36 Wenger GC, Tucker I. Using network variation in practice: identification of support network type. Health Soc Care Community 2002;10:28-35.

37 Prince M, Ferri CP, Acosta D, et al. The protocols for the 10/66 dementia research group population-based research programme. BMC Public Health 2007;7:165

38. Prince $\mathrm{M}$, Acosta $\mathrm{D}$, Chiu $\mathrm{H}$, et al. Dementia diagnosis in developing countries: a cross-cultural validation study. Lancet 2003;361:909-17.

39. Copeland JR, Dewey ME, Griffiths-Jones HM. A computerized psychiatric diagnostic system and case nomenclature for elderly subjects: GMS and AGECAT. Psychol Med 1986;16:89-99.

40. Hall KS, Hendrie HC, Brittain HM, et al. The development of a dementia screening interview in two distinct languages. Int $J$ Methods Psychiatr Res 1993;3:1-28.

41. Ganguli M, Chandra V, Gilby JE, et al. Cognitive test performance in a community-based nondemented elderly sample in rural India: the Indo-U.S. Cross-National Dementia Epidemiology Study. Int Psychogeriatr 1996;8:507-24.

42. World Health Organization. WHODAS Disability Assessment Schedule 2.0. 2001 http://www.who.int/classifications/icf/more whodas/en/

43. Taylor SE, Sherman DK, Kim HS, et al. Culture and social support: who seeks it and why? J Pers Soc Psychol 2004;87:354-62.

44. Kaplan D. V, Berkman BJ. Effects of Life Transitions on the Elderly. New Jersey: Merck, 2016. https://www.merckmanuals.com/en-ca/ professional/geriatrics/social-issues-in-the-elderly/effects-of-lifetransitions-on-the-elderly (accessed 20 Dec 2018).

45. Hershey DA, Henkens K. Impact of different types of retirement transitions on perceived satisfaction with life. Gerontologist 2014;54:232-44.

46. Civil Service College. Ethos. 2006 https://www.cscollege.gov.sg/ Knowledge/Ethos/Lists/issues/Attachments/10/ETHOS\%20IS01.pdf (accessed $18 \mathrm{Jul}$ 2017).

47. Ministry of Health. Action plan for successful ageing. $2016 \mathrm{https} / / /$ www.moh.gov.sg/content/moh_web/successfulageing.html (accessed 18 Jul 2017).

48. Ministry of Finance. Budget in Brief; Budget. 2018 https://www. singaporebudget.gov.sg/data/budget_2018/download/FY2018_ Budget in Brief ENG.pdf (accessed 31 Dec 2018).

49. Wenger GC. Social networks and gerontology. Rev Clin Gerontol 1996;6:285-93.

50. Wenger GC. Social networks and the prediction of elderly people at risk. Aging Ment Health 1997;1:311-20.

51. Fratiglioni L, Wang HX, Ericsson K, et al. Influence of social network on occurrence of dementia: a community-based longitudinal study. Lancet 2000;355:1315-9.

52. Teo P, Mehta K, Thang LL, et al. Ageing in Singapore: Service needs and the state. London: Routledge, 2006.

53. Kim C, Wu B, Tanaka E, et al. Association between a Change in Social Interaction and Dementia among Elderly People. Int J Gerontol 2016;10:76-80.

54. Li J, Theng YL, Foo S. Depression and Psychosocial Risk Factors among Community-Dwelling Older Adults in Singapore. J Cross Cult Gerontol 2015;30:409-22.

55. Alexopoulos GS, Meyers BS, Young RC, et al. Recovery in geriatric depression. Arch Gen Psychiatry 1996;53:305-12.

56. Murphy $\mathrm{E}$. The prognosis of depression in old age. $\mathrm{Br} J$ Psychiatry 1983;142:111-9. 